\title{
Captures
}

Figures, théories et pratiques de l'imaginaire

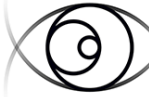

C A P T U R E S

\section{Entre les lignes}

\section{Jean-Marie Privat}

Volume 2, Number 2, 2017

Imaginaire de la ligne

URI: https://id.erudit.org/iderudit/1059767ar

DOI: https://doi.org/10.7202/1059767ar

See table of contents

Publisher(s)

Figura, Centre de recherche sur le texte et l'imaginaire

ISSN

2371-1930 (digital)

Explore this journal

Cite this article

Privat, J.-M. (2017). Entre les lignes. Captures, 2(2).

https://doi.org/10.7202/1059767ar

\section{Article abstract}

Les lignes d'écriture n'existent ordinairement que si elles tirent droit et se tiennent à équidistance les unes des autres. Ce dispositif graphique dessine un entre-deux où toute une série d'activités d'écriture ou de lecture signifiantes peuvent toutefois s'intercaler. Cette économie sémiotique et pragmatique, poïétique et politique de l'interligne, entre interdit et inter-dit, donne parfois corps à un investissement imaginaire graphique ou plastique étonnant et transgressif que cet article entend suivre et analyser.
Tous droits réservés @ Jean-Marie Privat, 2017

\section{(c) (i) $\Theta$}

This document is protected by copyright law. Use of the services of Erudit (including reproduction) is subject to its terms and conditions, which can be viewed online.

https://apropos.erudit.org/en/users/policy-on-use/
This article is disseminated and preserved by Érudit.

Érudit is a non-profit inter-university consortium of the Université de Montréal, Université Laval, and the Université du Québec à Montréal. Its mission is to promote and disseminate research.

https://www.erudit.org/en/ 
Version enrichie de cet article : http://revuecaptures.org/node/942

\section{Entre les lignes}

\section{Jean-Marie Privat}

Résumé :

Les lignes d'écriture n'existent ordinairement que si elles tirent droit et se tiennent à équidistance les unes des autres. Ce dispositif graphique dessine un entre-deux où toute une série d'activités d'écriture ou de lecture signifiantes peuvent toutefois s'intercaler. Cette économie sémiotique et pragmatique, poïétique et politique de l'interligne, entre interdit et inter-dit, donne parfois corps à un investissement imaginaire graphique ou plastique étonnant et transgressif que cet article entend suivre et analyser.

Written lines usually exist only if they lie straight and equidistant one from another. This graphical device opens up a space in between, where a series of scriptural activities may significantly take place. The semiotics of interlining at times incite a graphic investment of these blank areas which, through writings or readings, may surprise or transgress. Such marginal markings are the ones that this article attempts to study and to analyse.

Cet essai se voudrait une propédeutique illustrée à un fragment delinéalogie de l'écriture et de la lecture. II existe certes une bibliographie matérielle qui se préoccupe de la description formelle des livres, rares ou ordinaires. On trouve aisément des études nombreuses sur la page, la marge, le paragraphe. Sur la typographie et l'iconographie. Et si la ligne passionne les calligraphes, les plasticiens et les anthropologues (Ingold, 2011), elle n'est guère approchée comme une technologie de l'écrit. On se doute qu'a fortioril'interligne est généralement tenu pour une pure contrainte matérielle liée à l'ordre graphique, sans signifiance particulière. Or, pour pasticher Mallarmé, cet « unanime blanc » (1998 [1887]: 42) entre parfois en dialogie formelle et conceptuelle avec les autres signes du discours. S'entrouvrent alors dans cet entre-deux des horizons imaginaires qui peuvent se jouer de la domestication graphique et ensauvager le lieu et la formule C'est à un libre parcours dans cet univers faussement familier — entre poétique et politique de l'interligne — que nous invitons notre lecteur.

Écrire, c'est écrire sur un support matériel (pierre, papyrus, parchemin, papier, écran) sur lequel on décide de délimiter une surface d'inscription propre (bidimensionnelle, homogène, quadrangulaire, etc.) : « Je désigne par écriture l'activité concrète qui consiste sur un espace propre, la page, à construire un texte qui a pouvoir sur l'extériorité dont il a d'abord été isolé. » (Certeau, 1980: 234-235.) II reste à organiser un espace graphique (marges, lignes horizontales et verticales équidistantes et orthogonales, espacements typographiques et retraits divers 1 ), et comme on dit à régler sa feuille : 
« Régler I. Tracer à l'aide d'une règle, ou d'un appareil qui en tient lieu, des lignes droites parallèles sur une surface unie, le plus souvent sur du papier. Le soir, à l'étude, il tira ses bouts de manches de son pupitre, mit en ordre ses petites affaires, régla soigneusement son papier (Flaub., Mme Bovary, t. 1, 1857, p. 4). 》 (ATILF.)

Ces trois opérations séquentielles visent bien sûr à accueillir les signes (alphabétiques ou non) de l'écriture. C'est la dimension sémaphorique du dispositif, particulièrement de l'ensemble structuré et géométrisé des lignes qui, étymologiquement et concrètement, portent l'écrit. Le premier livre imprimé par Gutenberg à l'aide de caractères mobiles est d'ailleurs connu justement sous le nom de Bible latine à quarante-deux lignes (1455). Point d'écrit sans ligne donc, mais point de ligne non plus sans interligne 2 . C'est précisément cette alternance régulière des lignes et des interlignes qui constitue l'infrastructure formelle de la page, page d'écriture et page de lecture. Dans ce microsystème d'opposition discrète et binaire, le blanc de l'interligne s'oppose par principe au noir de la ligne (virtuelle ou tracée, puis écrite) comme le plein s'oppose par définition au vide interlinéaire. Tel une barrière à claire-voie, à plat et sans arrière-pays. Mais comme tout dispositif sémiotique, aussi élémentaire soit-il, il peut être transgressé de multiples manières.

\section{Écrire un silence}

La littérature connaît en fait deux façons également radicales de défaire la solidarité élémentaire de la ligne horizontale d'écriture et des espaces interlinéaires conjoints. La première serait la page blanche, rituellement blanche - comme une minute de silence graphique. C'est par exemple le cas de l'émouvante sobriété de la page 142 du livre de Maurice Cling, Un enfant à Auschwitz, qui ne saurait dire mieux l'indicible :

Le lecteur est prié d'excuser ce qui va suivre : une page blanche symbolique. Soixante-dix ans après cette nuit tragique, la douleur est aussi vive que jadis. Qu'il suffise de savoir que les matricules des « sélectionnés » furent appelés, que les camions bâchés arrivèrent et qu'ils disparurent avec leur chargement au coin du Block [...]. (2015 [1999]: 141.)

Une variante de ce silence typographique se retrouve dans l'autobiographie sensible d'Alice Rivaz,L'Alphabet du matin (2002). L'écrivaine clôt le récit d'une tragédie d'enfance dans une dialectique de l'achrome blanc qui voudrait lever l'encre pour coder le noir :

Et maintenant, que n'ai-je deux, trois, quatre pages blanches à ma disposition pour n'y rien écrire, et les glisser dans mon récit en manière de silence, de repos, de mesure pour rien, mais combien nécessaires, avant de retrouver mes puérils soucis, mes grands ou petits bonheurs de petite fille. (Rivaz, 2002: 167.)

À l'opposé de cette poétique expressive et commémorative de la page blanche, le romancier peut choisir de 
manifester son émotion par une page noire - comme si le deuil envahissait toute la page et la nuit obscurcissait les balises ordinaires du langage écrit. On pense bien sûr à l'imagination typographique exacerbée de Laurence Sterne. Sur une « dalle de marbre uni » posée sur sa tombe, les exécuteurs testamentaires et amis d'un malheureux personnage qui répond au nom shakespearien de « Yorick » font graver ces « trois simples mots » : « Hélas! pauvre YORICK! » (Sterne, 2004 [1762]: 59-60.) En guise de prolongement suit un faire-part humoristique, aigre-doux, sous la forme d'une pleine page recto verso passée au noir le plus funèbre et bordée de strictes marges blanches (61-62).

\section{Écrire entre}

Si l'interlignage peut-être négatif (absent) — comme on dit dans le langage des typographes —, on voit bien qu'inversement cet espace vierge constitue de facto un lieu matériel disponible 3 et tentant pour toute espèce d'insertion interlinéaire. La typographie devient alors topographie. C'est par exemple la traduction interlinéaire des polyglottes ou la glose interlinéaire des philologues d'autrefois 4 . C'est encore le cryptage des lettres diplomatiques ou des correspondances codées ${ }^{-}$. Ce peut-être aussi, on s'en doute, l'espace du crime ou de la fraude (à l'encre visible ou invisible), ces interlignes qui sont alors, comme l'écrit emphatiquement le fidèle Las Cases, «les véritables archives de la bassesse, du mensonge et de la vilenie » :

On trouva $[\ldots]$ une longue lettre d'une des femmes de la princesse Pauline. Cette volumineuse lettre s'exprimait fort mal sur la princesse et ses sœurs, et ne parlait de cet homme (c'était l'Empereur) que sous les plus mauvaises couleurs. On n'avait pas cru que ce fût assez, on en avait raturé une partie, et interligné d'une main étrangère, pour y faire arriver Napoléon lui-même de la manière la plus scandaleuse; et à la marge, et de la main de l'interligneur, il y avait : Bon à imprimer. Quelques jours de plus, probablement ce petit libelle allait voir le jour. (Las Cases, 1823: t. I, 327-328.)

Écrire ou inscrire entre comme les motifs des manuscrits médiévaux enluminés, motifs qui s'originent dans la marge et viennent se glisser entre les lignes d'écriture que trace-dessine le scribe (plume d'oiseau merveilleux, trompe d'animal fabuleux, arabesque endiablée, queue de lettre ou herbe ornée, etc.). Comme si la page interlignée, ensauvagée à la marge et par la marge, ne s'alignait pas aussi continûment sur les injonctions d'un quadrillage graphique strict, ce lieu planifié et programmé que la modernité de l'écrit va réputer bientôt comme idéologiquement « désensorcelé des ambiguïtés du monde » (Certeau, 1980: 234-235). C'est, plus près de nous, l'espace de fortune et d'infortune de l'écrivain durement privé de papier :

Dans le temps de la Régence, M. de Voltaire fut mis à la Bastille et y resta un an; il y fit une grande partie de La Henriade, mais comme il n'avoit ny papier ny plume, il l'écrivit dans les interlignes d'un petit livre qu'on luy avoit laissé, et il se servit d'un crayon. (Cideville cité par Niderst, 1970: 464.) 
C'est enfin le lieu de la pédagogie à l'ancienne et où le papier est rare et cher :

Aux $\mathrm{XVI} \mathrm{I}^{\mathrm{e}}$ et $\mathrm{XVII}{ }^{\mathrm{e}}$ siècles, les textes que le professeur a mis à son programme de prélection sont publiés par ses soins à partir d'une édition savante et imprimés localement sur des « feuilles classiques », petits livrets bon marché, de facture typographique grossière, qui ménagent des interlignes et des espaces blancs destinés à recueillir les notes de l'élève. (Chervel, 1997: 14.)

\section{S'écrire entre}

Sur un mode plus libre et plus créatif, les interlignes sont les espaces où s'inscrivent parfois les corrections ou ajouts dits précisément interlinéaires. Or, ces écritures interlinéaires sont par exemple constitutives des Essais de Montaigne, du moi le plus privé de Montaigne en ses successives couches rédactionnelles (2016 [1588-92]: 492v). On le pressent, si les additions interlinéaires sont à la fois manuscrites et discontinues, c'est que cet espace blanc peut être conçu et perçu comme une bande de défilement qui s'offre aux imaginaires de l'écriture. À ses inter-dits tragiques, à ses ellipses poétiques, à ses beautés bibliophiliques aussi. Pensons aux conseils que son éditeur donne à Elie Wiesel : « [...], la mort de mon père, la libération. Pourquoi ne pas les inclure [...]? - Trop personnels, trop intimes, ils doivent rester entre les lignes. » (Wiesel, 2007 [1958]: 15.) Songeons aux innombrables suggestions plastiques et ressources sémiotiques que les écrivains ont tiré de l'intra-linéaire, à l'image du poème « Le cageot », de Francis Ponge :

À mi-chemin de la cage au cachot, la langue française a cageot, simple caissette à claire-voie vouée au transport de ces fruits qui de la moindre suffocation font à coup sûr une maladie. (1982: 32.)

Partageons enfin un instant la réflexion de Paul Valéry sur le jeu décisif des lignes et des interlignes dans l'esthétique du livre (la bibliophilie) et la pragmatique de la lecture (le souci du lecteur) :

Le texte vu, le texte lu sont choses toutes distinctes, puisque l'attention donnée à l'une exclut l'attention donnée à l'autre. Il y a de très beaux livres qui n'engagent pas à la lecture, belles masses de noir pur sur champ très pur, mais cette plénitude et cette puissance de contact obtenues aux dépens des interlignes [...] ne sont pas sans peser sur le lecteur, et sans paraître un peu trop archaïques. La littérature moderne ne s'accommode pas de ces formes compactes et comme gorgées de caractères [...]

Un livre est matériellement parfait quand il est doux à lire, délicieux à considérer; quand enfin le passage de la lecture à la contemplation, et le passage réciproque de la contemplation à la lecture sont très aisés et correspondent à des changements insensibles de l'accommodation visuelle. Alors les noirs et les blancs sont des repos l'un de l'autre, l'œil circule sans effort dans son domaine bien disposé, en apprécie l'ensemble et les détails et se sent dans les conditions idéales de son fonctionnement. (1936: 19-21.) 
Ainsi vient sinon s'inscrire du moins s'immiscer un effet de fiction dans les espaces interstitiels de laraison graphique (Goody, 1979). L'écrit et l'écrivain y composent et y proposent une plus-value de la signifiance, non plus seulement une sémaphorie de la ligne, mais un « commun alignement » de la prose, pour reprendre le syntagme de Mallarmé (2003 [1897]: 216). C'est bel et bien une sémiophorie de l'interligne 6 . Victor Hugo le disait en son langage, à propos d'un autre type de blanc : «L'oubli n'est autre chose qu'un palimpseste. Qu'un accident survienne, et tous les effacements revivent dans les interlignes de la mémoire étonnée. » (Hugo, 2012 [1869]: 449.)

\section{Comme dans une fente...}

Certes l'interligne est en principe un espace vierge, franc et probe que sa blancheur défend :

Elle [Mme de Vins] m'a écrit une lettre vive et fort jolie. Elle se plaint de mon silence [...]. Je n'ai jamais vu un fagot d'épines aussi révolté. Je lui fais réponse, et me réjouis qu'elle se soit mise à être tendre et à parler de la jalousie, autrement qu'en interligne 7 [...]. (Sévigné, 1973-78 [lettre à Mme de Grignan du 3 nov. 1675]: t. II, 149.)

Mais il est d'expérience commune que l'on puisse (ou doive) lire entre les lignes. Une « certaine finesse d'interprétation » ou d'imagination est en effet requise, comme dit Proust (1987 [1921]: 431). On est assez loin d'un espace purement typographique, aussi anodin qu'inaperçu. La sémantique est au contraire peu ou prou une mantique... L'écrivain peut jouer en plusieurs façons de cette signifiance potentielle de l'interligne et susciter une sorte de rêverie (ou de panique) interlinéaire, par personnages interposés.

Madame Bovary offre de ce point de vue un exemple assez passionnant de la conscience graphique interlinéaire, ses charmes et ses larmes. Ainsi, quand Charles vient de découvrir la lettre funeste où Emma explique son geste suicidaire et que l'officier de santé s'enquiert de la pharmacopée contre les poisons, les lignes dansent sous ses yeux — comme une inquiétante danse des mots (des morts) :

Charles voulut feuilleter son dictionnaire de médecine; il n'y voyait pas, les lignes dansaient. — Du calme! dit l'apothicaire. II s'agit seulement d'administrer quelque puissant antidote. Quel est le poison?

Charles montra la lettre. C'était de l'arsenic. (Flaubert, 1971 [1857]: 324.)

Ce brouillage émotionnel de la lecture qui déplace les lignes et déstabilise les interlignes est (relativement) mieux maîtrisé par Emma quand elle découvre, avec la lucidité que peut conférer une extrême inquiétude, le papier de la saisie de ses biens :

En rentrant chez elle, Félicité lui montra derrière la pendule un papier gris. Elle lut : 
«En vertu de la grosse, en forme exécutoire d'un jugement... »

Quel jugement? La veille, en effet, on avait apporté un autre papier qu'elle ne connaissait pas; aussi futelle stupéfaite de ces mots :

"Commandement de par le roi, la loi et la justice, à Mme Bovary... »

Alors, sautant plusieurs lignes, elle aperçut :

«Dans vingt-quatre heures pour tout délai. » - Quoi donc? « Payer la somme totale de huit mille francs. » Et même il y avait plus bas : "Elle y sera contrainte par toute voie de droit, et notamment par la saisie exécutoire de ses meubles et effets. [...] »(298-299.)

Certes, cette dé-linéarisation de la lecture du document sert la logique narrative de la scène et la peinture psychologique du personnage. Mais ici Emma passe les lignes plus qu'elle ne lit entre les lignes. Or, rêver entre les lignes est une manière d'être et de faire propre à l'imaginaire (et à l'esthétique) du roman de Flaubert. Cette rêverie est tantôt un embrayeur d'imaginaire pour tel ou tel personnage de la fiction, tantôt elle est une interruption de la monotone linéarité, une suspension discrètement offerte au lecteur. Voici Emma au comble de l'ennui conjugal et de la solitude existentielle :

Elle s'était acheté un buvard, une papeterie, un porte-plume et des enveloppes, quoiqu'elle n'eût personne à qui écrire; elle époussetait son étagère, se regardait dans la glace, prenait un livre, puis, rêvant entre les lignes, le laissait tomber sur ses genoux. Elle avait envie de faire des voyages ou de retourner vivre à son couvent. Elle souhaitait à la fois mourir et habiter Paris. (62.)

La posture (la pose) romantique de l'héroïne dit son insatisfaction (sonbovarysme) à suivre le fil du récit et son échappée interlinéaire dans une autre fiction, une forme d'autofiction cette fois. Les lignes ne sont alors que prétextes vite abandonnés pour la fabrique d'un chimérique intertexte intra-linéaire, un mirage de la page. Dans une autre page, entièrement biffée celle-là de la version définitive du texte, Flaubert imagine qu'Emma s'ingénie à écrire à Léon de longues lettres où passent des fleurs et des nuages, des peupliers qui se balancent au vent et des longs désirs de voyage... L'épistolière amoureuse s'efforce en fait d'aviver son inspiration « à tous les secours de la Poésie à chaque ligne lettre entre les lignes, tout en éerivant, rêvantà lui, elle aurait voulu être un papier qui palpiterait " (Flaubert, c2003 [1851-57]: vol. 6, P 43v). Cette occurrence manifeste une dialectique de l'écriture « poétique » et de l'intime songerie mais aussi, entre les lignes, l'irruption de l'image de l'amant et le fantasme érotique d'une communication écrite qui serait somaphorique ${ }^{8}$.

Cette fantasmagorie d'une lettre vive - comme unpunctum d'oralité pure, à corps présents - se retrouvait déjà, fût-ce sur le mode du libertinage égotiste, chez Rodolphe, l'autre amant, dans une page d'une série de brouillons antérieure, elle aussi entièrement biffée : 
[...] et les aventures revenaient — des visages oubliés, lui souriaient.— des tableaux des situations. il revoyait comme dans une fente entre les lignes. Sentiment.— mais qq unes de complètement perdues oubliées. Ainsi \& qu'il cherchait. Ainsi ceci au crayon oui tantôt à l'heure dite [...] il rêva sans pouvoir trouver - et y renonça faisant des efforts \& ne pouvant trouver toute-sa jeunesse amoureuse revenait palpiter sur ees pages oubliées. confondues. Cependant des visages figures oubliés lui souriaient réapp. tout à coup dans l'intervalle des lignes $\stackrel{9}{ }$. (Flaubert, c2003 [1851-57]: vol. 4, f 183v.)

Même si Flaubert n'a pas retenu cette version pour la publication, il reste qu'un tel imaginaire scriptural (de Gustave, ou d'Emma ou de Rodolphe?) joue le rôle d'une plaque sensible où vient s'imprimer une mémoire interlinéaire affective et affectueuse, entre rêverie érotique et hallucination sémiotique : l'intervalle des lignes comme « fente $\underline{10}$ ». L'ordre de l'écrit est ainsi à la fois écran et écrin à la puissance imageante spontanée. L'écrit s'entrouvre alors sur l'inter-scrit comme ou sur un corps de femme, les visages sourient comme des revenants reconnaissants perdus de vue et sur le «blanc du papier » (Freud, 1970 [1916]: 124) se frayent les émois du moi amoureux de jadis $\frac{11}{1}$. Cet imaginaire scopique de l'interligne est à la fois le comble ironique de la raison graphique (sa béance est sa créance) et l'expression même de l'intime liberté du lecteur.

Aussi cet entre-texte est-il un outre-texte où s'établissent des liens dialogiques entre le scripteur d'autrefois et la recherche interlinéaire du temps retrouvé par son lecteur d'aujourd'hui lui-même.

\section{Et il y avait ici un intervalle entre les lignes}

Dans le corpus romanesque que nous avons choisi de privilégier, il reste un dernier cas de figure où se confondent cette fois l'espace scriptural de l'interligne et l'espace mental du scripteur, et peut-être même le personnage, l'auteur et son lecteur :

C'était l'époque où le père Rouault envoyait sa dinde, en souvenir de sa jambe remise. Le cadeau arrivait toujours avec une lettre. Emma coupa la corde qui la retenait au panier, et lut les lignes suivantes :

«Mes chers enfants,

J'espère que la présente vous trouvera en bonne santé [...]. J'ai eu un malheur à ma charreterie, dont la couverture, une nuit qu'il ventait fort, s'est envolée dans les arbres. La récolte non plus n’a pas été trop fameuse. Enfin, je ne sais pas quand j'irai vous voir. Ça m'est tellement difficile de quitter maintenant la maison, depuis que je suis seul, ma pauvre Emma! »

Et il y avait ici un intervalle entre les lignes², comme si le bonhomme eût laissé tomber sa plume pour rêver quelque temps.

«Quant à moi, je vais bien, sauf un rhume que j'ai attrapé l'autre jour à la foire d'Yvetot [...]. »

« II me fait deuil de ne pas connaître encore ma bien-aimée petite-fille Berthe Bovary [...]. » 
Adieu, mes chers enfants. Je t'embrasse, ma fille; vous aussi, mon gendre, et la petite, sur les deux joues. » (Flaubert, 1971 [1857]: 175-176.)

« [Q]ue ne ferme-t-on les yeux à rêver? » se demandait Mallarmé, las du « va-et-vient successif incessant du regard, une ligne finie, à la suivante, pour recommencer » (2003 [1897]: 226). Le bonhomme Rouault — le père d'Emma - n'est certes pas mû par les mêmes considérations esthétiques que le subtil poète en quête d'un « espacement de la lecture » (Mallarmé, 1998 [1897]: 391). Le récit n'en mentionne pas moins son geste de scripteur (un stéréotype de la posture romantique) et sa rêverie lamartinienne au subjonctif plus-que-parfait, si proches des méditations lyriques prêtées passim à Emma... Si l'émotion paternelle n'est pas feinte, c'est bien le travail d'écriture comme processus rédactionnel (s'enquérir des siens, informer sur soi, témoigner de son affection, etc.) qui est interrompu par une sorte de digression psychique. Ce blanc momentané de l'écriture est toutefois marqué par une ligne pleine qui de facto dénie matériellement ce qu'elle assume sémantiquement. Mais, même si l'intervention méta-scripturale du narrateur flaubertien («Et il y avait ici un intervalle [...] ») n'est en rien mimétique et même contredit son propre signifié verbal, elle porte bel et bien sur la disposition du signifiant typographique. Elle signifie aussi que l'épistolier normand n'est pas tout à fait à l'aise avec les convenances scripturales et la continuité formelle de l'écriture. La ligne ne se contente pas d'ailleurs de commenter son interligne puisqu'elle suggère au lecteur de voir en quelque façon la version manuscrite de la lettre écrite par un père si solitaire à sa fille si absente. Or, le déictique spatial « ici » ancre-encre le discours du narrateur dans la situation d'énonciation et focalise expressément le champ de vision du lecteur sur une soudaine discontinuité interlinéaire. Comme si une fois encore la vacance scripturale était seule à même de signifier un certain malaise existentiel (« ma pauvre Emma », « il me fait deuil », « adieu ») $\underline{13}$. II n'est d'ailleurs pas exclu que cet investissement contre et par l'écriture soit aussi une mise en abyme de Flaubert lui-même au travail, et la rêvasserie sur ses propres phrases...

\section{Le texte, la texture, l'aventure}

On ne saurait donc dissocier par principe la textualité linguistique (le texte) de la matérialité de l'écrit (la texture) sans s'exposer à ignorer les éventuels processus de signifiance interlinéaire, en lecture comme en écriture 14 . C'est même précisément parce que l'interligne est le constituant formel et impératif d'une ligne d'écriture que l'exploitation graphique et l'exploration poïétique de ce non-lieu sont à la fois proscrites et leurs transgressions plus ou moins hétérotextuelles... pré-scrites. II arrive même, comme nous l'avons esquissé avec Flaubert, que l'esthétique (et l'éthique) du « livre sur rien » trouve fugacement son lieu et sa formule dans la plénitude immatérielle de l'interligne :

Ce qui me semble beau, ce que je voudrais faire, c'est un livre sur rien, un livre sans attache extérieure [...], un livre qui n'aurait presque pas de sujet ou du moins où le sujet serait presque invisible, 
si cela se peut. Les œuvres les plus belles sont celles où il y a le moins de matière [...]. (Flaubert, 2017 [lettre à Louise Colet du 16 jan. 1852].)

À vrai dire, il y a un puissant imaginaire culturel (et inconscient) de l'interstice et de l'intervalle 5 , et en strict régime littératien de cet entre-ligne $\frac{16}{6}$ qu'il ne convient pas — sous le regard dudroit - d'outrepasser. C'est ainsi que jusque dans notre langue même s'immiscent des veuves et des orphelines, s'intercalent des lames et des sillons. Il serait en effet intéressant de comprendre l'imaginaire du jargon (patriarcal?) des typographes pour qui une ligne veuve « est la dernière ligne d'un paragraphe qui se trouve être la première ligne d'une page » et une ligne orpheline « la première ligne d'un nouveau paragraphe lorsqu'elle au bas de la page » (Cormier: 31). Bien sûr, on essaye généralement d'« [é]viter les veuves et orpheline[s] », précisent les lexiques professionnels (Cormier: 67). Les typographes savent aussi qu'une interligne est une fine lame de métal de longueur égale à celles des lignes de composition qui sert à séparer matériellement les lignes; cette lame intercalaire détermine ce faisant l'épaisseur de l'interligne. Et les dictionnaires les plus élémentaires nous apprennent qu'interligne désigne tout aussi bien l'espace typographique que le texte interlinéaire lui-même...

Enfin, la tardive extension polysémique de ses emplois ouvre le motinterligne à des correspondances scripturales fascinantes entre la page (ses lignes d'écriture) et le pagus (la vigne et ses rangées de ceps) 17 , entre le corps du texte et l'articulation du corps 18 . Aussi ne s'étonnera-t-on pas vraiment des cheminements linéaires dévoyés et des excursus interlinéaires dérivant des imaginaires des ensauvagés graphiques des temps anciens et des temps nouveaux. Tel ce Nicolas Cirier, entre folie plastique et insoumission poéticolinéaire (Cirier, 1840). Tels Reinhold Metz et son fouillis littéral ou Constance Schwartzlin-Berberat et ses accolements linéaires $\frac{19}{}$. Tels enfin ce musicien qui insuffle ces notes vives dans les interlignes d'une page de musique et ce jongleur, son compère, qui lance par-delà les lignes ses balles multicolores et virevoltantes. Une fantaisie musicale et une expressivité gestuelle, une oralité charmeuse et une corporalité joueuse, entre les lignes.

1. Pour un aperçu anthropologique des usages de la règle dans les cultures de la ligne droite, "icône de la modernité », voir le chapitre d'Ingold intitulé « Utiliser une règle » (2011: 208-218).

2. L'interligne est stricto sensu « l'espace située entre le haut des minuscules de deux lignes consécutives [...]. Les ascendantes et les descendantes ${ }^{\star}$ des caractères débordent à l'intérieur de cette espace ${ }^{\star \star}$ mais ne diminuent guère l'écart entre les lignes. En général, si l'on utilise, pour séparer les lignes, la valeur du corps, le résultat est confus et difficile à lire. Pour cette raison, on écarte les lignes d'une valeur supérieure à celle du corps. Cet espacement est proportionnel au corps du caractère d'un ou deux points. C'est ainsi que pour des caractères de corps 12, les lignes seront généralement séparées de 13 ou 14 points. 》 (Cormier, p. 32.$)$ * "L'ascendante d'un caractère est la hauteur de celui-ci qui se trouve au-dessus de sa ligne de base. La descendante est quant à elle, la hauteur du caractère au-dessous de la ligne de base. » (Cormier, p. 32, n. 2.) ** « On remarquera qu'en typographie le mot "espace" est au féminin quand il s'agit du caractère. Cela vient du fait qu'à l'origine, il s'agissait d'une petite cale métallique placée par les typographes pour séparer les mots entre eux. » (Cormier, p. 32, n. 3.)

3. Voltaire, un exemple entre mille autres auteurs, admire particulièrement la typographie interlinéaire des anglais : « Je reçois dans ce moment, mon cher ami, les trois volumes de l'histoire de Charles-Quint que Mr Robertson m'envoie. Le livre est imprimé avec des interlignes lesquelles sont doubles à chaque paragraphe. Tous les livres sont imprimés ainsi en Angleterre. Rien ne contente 
plus les yeux; rien ne délasse plus le lecteur. » (Voltaire, 1952 [1770]: 211.)

4. Mérimée dans la deuxième édition de ses Mélanges historiques et littéraires (1868 [1855]) se moque de ces "doctes désœuvrés 》 qui, parcourant un traité de scolastique ou tout autre « fatras du moyen âge », ont aperçu dans les interlignes les traces d'un ouvrage plus ancien mal effacées et lisibles encore pour quelques yeux érudits. II s'interroge alors sur le palimpseste interlinéaire : «Pourquoi ne retrouverait-on pas dans des interlignes une comédie de Ménandre ou les livres qui nous manquent de TiteLive? [...]. Tout récemment, dans un évangile syriaque du Musée britannique, on a constaté comme l'ombre d'un manuscrit trèsancien. » (362-363.) Béroalde de Verville au début du XVII siècle ironisait déjà, lui, contre l'inflation savantes des « gloses et interlignes pédantines » (75).

5. Jadis, pour éviter toute fraude et additions faites après coup dans les écritures administratives et financières, le législateur prévoyait que « les mentions de dépôts, les inscriptions et transcriptions, sont faites sur les registres, de suite, sans aucun blanc ni interligne, à peine, contre le conservateur, de mille à deux mille francs d'amende, et des dommages et intérêts des parties, payables aussi par préférence à l'amende » ([s. a.], 1804: 404). C'est même un terme de droit rapporté par Littré (1886: 203) : « Écrit entre les lignes. Les mots surchargés, interlignés ou ajoutés seront nuls, Loi du 25 ventôse an XI, art. 16 ».

6. Sur l'opposition sémaphore (dispositif technique dont le rôle se limite à être un support matériel de messages, linguistiques ou non, tel les sémaphores qui régulent la circulation routière - par exemple) et sémiophore (configuration signifiante qui constitue en soi et par soi un microsystème de signes spécifiques), on peut se reporter à Privat (2013).

7. $\quad$ [Nous soulignons.]

8. Emma, faute de mieux, prend plaisir à toucher d'un doigt fabulateur le monde cartographié, si proche et si loin en sa mimésis codée : «Elle s'acheta un plan de Paris, et, du bout de son doigt, sur la carte, elle faisait des courses dans la capitale. Elle remontait les boulevards, s'arrêtant à chaque angle, entre les lignes des rues, devant les carrés blancs qui figurent les maisons. " (Flaubert, 1971 [1857]: 59.) Sa puissance d'imagination graphique est nourrie de tous ses romanesques désirs : "Les yeux fatigués à la fin, elle fermait ses paupières, et elle voyait dans les ténèbres se tordre au vent des becs de gaz, avec des marchepieds de calèches, qui se déployaient à grand fracas devant le péristyle des théâtres. » (59.)

9. La version publiée est décevante de positivisme psychologique, y compris en son facile cynisme scripturaire : « II alla chercher dans l'armoire, au chevet de son lit, une vieille boîte à biscuits de Reims où il enfermait d'habitude ses lettres de femmes, et il s'en échappa une odeur de poussière humide et de roses flétries [...]. Enfin il lut de ses lettres [...]. II voulut revoir les longues, celles d'autrefois; pour les trouver au fond de la boîte, Rodolphe dérangea toutes les autres; et machinalement il se mit à fouiller dans ce tas de papiers et de choses [...]. II examinait les écritures et le style des lettres [...]. Elles étaient tendres ou joviales, facétieuses, mélancoliques [...]. À propos d'un mot, il se rappelait des visages, de certains gestes, un son de voix; quelquefois pourtant il ne se rappelait rien. » (Flaubert, 1971 [1857]: 206.) Gisèle Séginger (1997: 100-103) a analysé comment dans les scénarios d'écriture du roman les ratures « explosent » dans l'interligne supérieur puis inférieur ou « s'enchaînent en cascade ». Ces liens verticaux ou en diagonale finissent par dessiner de véritables « arabesques verbales » et participent concrètement (graphiquement et spatialement) de la fabrique du texte.

10. On connaît le jeu de mots érotico-graphique sur l'In-fente : «L'Infante seule était plus belle [...]. » (Perrault, 1981 [1694]: 100.) L'écrivain lit avec délectation les contes de Perrault à l'époque de la rédaction de Madame Bovary : « J'ai lu ces jours-ci les contes de fées de Perrault; c'est charmant, charmant. Que dis-tu de cette phrase : "La chambre était si petite que la queue de cette belle robe ne pouvait s'étendre". Est-ce énorme d'effet, hein? » (Flaubert, 2017 [lettre à Louise Colet du 16 déc. 1852].)

11. Freud emploie l'expression lorsqu'il compare à des fins pédagogiques les effets de la censure politique (le caviardage des journaux) à la censure du surmoi. Le lecteur est alors à l'affût, comme Rodolphe en quête d'anamnèse : « Sur ces espaces blancs devaient figurer les passages [...] les plus intéressants, les "meilleurs passages”. » (Freud, 1970 [1916]: 124.)

12. Dans une version préparatoire l'écrivain hésitait entre sur la formulation : « intervalle entre les lignes ", "intervalles entre deux lignes » ou peut-être même « intervalle entre les deux lignes » (Flaubert, c2003 [1851-57]: vol. 4, fo 38v). La rature est dans l'interligne, comme il se doit :

- il y avait ici un intervalle

deux*

entre les lignes, comme si le bonhomme eût laissé tomber

sa plume, pr rêver qque temps (Flaubert, c2003 [1851-57]: vol. 4, f ${ }^{\circ} 38 \mathrm{v}$ )

13. Quelques lignes plus loin, une fois la lettre lue, on retrouve à la fois la matérialité des pratiques d'écriture populaire et les songes de contes de fées, avant le mariage... : «Emma resta quelques minutes à tenir entre ses doigts ce gros papier [...]. On avait séché l'écriture avec les cendres du foyer, car un peu de poussière grise glissa de la lettre sur sa robe, et elle crut presque apercevoir son père se courbant vers l'âtre pour saisir les pincettes. Comme il y avait longtemps qu'elle n'était plus auprès de lui, sur l'escabeau, dans la cheminée, quand elle faisait brûler le bout d'un bâton à la grande flamme des joncs marins qui pétillaient!... Elle se rappela des soirs d'été tout pleins de soleil. Les poulains hennissaient quand on passait, et galopaient, galopaient... » (177.)

14. Si nous devions situer notre approche, ce serait du côté des problématiques dites « intégrationnistes » qui, en l'espèce, ne sauraient faire " abstraction du caractère concret des activités qui établissent les valeurs sémiologiques » (Harris, 1993: 361). Pour un cadrage théorique de cette approche, voir le dossier «Écriture(s)» des Actes sémiotiques (2016) et particulièrement les contributions d'Isabelle Klock-Fontanille et de Sybille Krämer. 
15. Nous avons ici largement privilégié des corpus en prose pour travailler à nous dé-familiariser d'avec l'objet quotidien (comme disent les anthropologues), à estranger (comme disaient les formalistes russes) notre perception spontanée des codes scripturaux, et nous ouvrir à d'autres perspectives herméneutiques.

16. Le langage juridique d'autrefois désignait par le terme d'entre-ligne cet espace — où l'on ne devait légalement écrire.

17. Le Trésor de la langue française recense cette acception : «Interligne, subst. [...] a) AGRIC. Espace qui sépare deux sillons, deux rangées de plantes dans un champ. [...] L'enherbement d'un interligne sur deux est adopté dans certains vignobles girondins [...]. : (ATILF.) Michel Melot nous rappelle, quant à lui, que « la page et le pays ont une même étymologie : pagus. L'image du champ labouré par l'écriture est explicite dans le genre d'écriture dite "en boustrophédon", où les lignes se succèdent alternativement de gauche à droite puis de droite à gauche, comme le font la charrue et la loi gravée sur le grand mur de Gortys, ancien palais crétois. 》 (2006: 122-123.)

18. «MÉDECINE / - Interligne (articulaire). Espace qui sépare les deux éléments d'une articulation. Interligne radio-carpien [entre le radius et le carpe]. » (ATILF.)

19. Les «illuminations » de Reinhold Metz et les « pulsions graphiques » de Constance Schwartzlin-Berberat sont présentées par Michel Thévoz (1989: 59-80; 109-118).

\section{Bibliographie}

[s. a.]. 1804. Code civil des Français. Paris : Imprimerie de la République, 579 p.

ATILF. [s. d.]. «Interligne ». TLFi : Trésor de la langue Française informatisé Nancy : Université de Lorraine; CNRS. <http://stella.atilf.fr/>.

ATILF. [s. d.]. «Régler ». TLFi : Trésor de la langue Française informatisé Nancy : Université de Lorraine; CNRS. <http://stella.atilf.fr/>.

Béroalde de Verville, François. 2006 [1617]. Le Moyen de parvenir, édition de Michel Renaud, préface de Michel Jeanneret. Paris : Gallimard, «Folio classique », 592 p.

Certeau (DE), Michel. 1980. L'invention du quotidien. Arts de faire. Paris : Union générale d'éditions, « 10 / 18 », t. 1, 374 p.

CHERVEL, André et Marie-Madeleine Compère. 1997. «Les humanités dans l'histoire de l'enseignement du français ». Histoire de l'éducation, vol. 74, no 1, p. 5-38.

CIRIER, Nicolas. 1840. L'apprentif administrateur, pamphlet pittoresque (!), littérario-typographicobureaucratique, pouvant intéresser toute personne employée, employable, ex-employée / par quelqu'un de cette dernière catégorie. Paris : Cirier, $72 \mathrm{p}$. <http://gallica.bnf.fr/ark:/12148/bpt6k105066w/f1.image.r=litt\%C3\%A9rario-typographico-bureaucratique>.

CLING, Maurice. 2015 [1999]. Un enfant à Auschwitz, préface de Yannis Thanassekos, éd. rev. et corr. Ivry-surSeine : Éditions de l'Atelier; Éditions Ouvrières, 237 p.

CoRmier, Serge. 2003. L'Écrit et les normes typographiques. Nantes : Université de Nantes, 100 p. $<\underline{\text { http://www.irem.sciences.univ-nantes.fr/telecharge/ecrit.pdf }>\text {. }}$

Flaubert, Gustave. 1971 [1857]. Madame Bovary. Mceurs de province, introduction et notes de Claudine Gothot-Mersch. Paris : Garnier Frères, « Classiques Garnier », 469 p.

FLAUBERT, Gustave. 2003 [1851-1857]. Les manuscrits de Madame Bovary. Édition intégrale sur le web, 
sous la dir. de Danielle Girard et Yvan Leclerc. Rouen : Bibliothèque de Rouen. <http://www.bovary.fr/>. FREUD, Sigmund. 1970 [1916]. Introduction à la psychanalyse. Paris : Payot, " Petite Bibliothèque Payot », $443 \mathrm{p}$.

Goody, Jack. 1979. La raison graphique. La domestication de la pensée sauvage. Paris : Minuit, 274 p. HarRIS, Roy. 1993. «Écriture et langage», dans La sémiologie de l'écriture. Paris : CNRS, « Langage ». Hugo, Victor. 2012 [1869]. L'Homme qui rit. Paris : Pocket, 761 p.

INGOLD, Tim. 2011. Une brève histoire des lignes, traduit par Sophie Renaut. Bruxelles : Zones sensibles, $256 \mathrm{p}$.

KLock-FontAnILle, Isabelle. 2016. «Repenser l'écriture. Pour une grammatologie intégrationnelle». Actes sémiotiques, no 119. <http://epublications.unilim.fr/revues/as/5623>.

KRÄMER, Sybille. 2016. «Entre discursivité et iconicité, un nouveau regard sur les écritures». Actes sémiotiques, no 119. <http://epublications.unilim.fr/revues/as/5628>.

LAS CASES (DE), Emmanuel. 1823. Mémorial de Sainte-Hélène, ou Journal où se trouve consigné, jour par jour, ce qu'a dit et fait Napoléon durant dix-huit mois. Paris : Las Cases, $8 \mathrm{t}$.

LITTRÉ, Émile. 1886. «Interligné », dans Dictionnaire de la langue française. Supplément Paris : Hachette, p. 203.

Mallarmé, Stéphane. 1998 [1887]. «Une dentelle s'abolit... », dans CEuvres complètes, Poésies, édition présentée, établie et annotée par Bertrand Marchal. Paris : Gallimard, «Bibliothèque de la Pléiade », p. 4243.

MalLARMÉ, Stéphane. 1998 [1897]. «Observation relative au poème "Un coup de Dés jamais n'abolira le Hasard" », dans CEuvres complètes. Un coup de dés jamais n'abolira le hasard édition présentée, établie et annotée par Bertrand Marchal. Paris : Gallimard, « Bibliothèque de la Pléiade », t. 1, p. 391-392.

Mallarmé, Stéphane. 2003 [1897]. «Divagations », dans CEuvres complètes, édition présentée, établie et annotée par Bertrand Marchal. Paris : Gallimard, «Bibliothèque de la Pléiade », t. 2, p. 79-277.

Melot, Michel. 2006. «Au pays de la page», dans Livre, préface par Régis Debray. Paris : L'œil neuf, « L'âme des choses », p. 121-137.

MérImÉE, Prosper. 1868 [1855]. Mélanges historiques et littéraires, 2e édition. Paris : Michel Lévy, 382 p. MontalGne (DE), Michel. 2016 [1588-1592]. Les Essais, exemplaire de Bordeaux. Paris : L’Angelier, 496 p. NIDERST, Alain. 1970. «"Traits, notes et remarques" de Cideville (suite) ». Revue d'Histoire littéraire de la France, vol. 70, no 3, mai-juin, p. 455-471.

Perrault, Charles. 1981 [1694]. «Peau d'âne », dans Contes, édition présentée et annotée par Jean-Pierre Collinet. Paris : Gallimard, «Folio classique », p. 98-115.

PONGE, Francis. 1992. Le parti pris des choses, suivi de Proêmes. Paris : Gallimard, «Poésie », 221 p. Privat, Jean-Marie. 2013. «La piscine ou de l'écrit sans écriture... », dans Jean-François DiANA (dir.), 
Spectacles sportifs, dispositifs d'écritures. Nancy : Presses universitaires de Nancy, «Questions de communication. Série actes, 19 », p. 21-28.

Proust, Marcel. 1987 [1921]. À la Recherche du temps perdu. Le Côté de Guermantes, Il. Paris :

Flammarion, «GF », 480 p.

Rivaz, Alice. 2002 [1968]. L'Alphabet du matin. Vevey (Suisse) : L'Aire bleue, 271 p.

SÉGINGER, Gisèle. 1997. «Chorégraphies scripturales de Flaubert. De l'inspiration à l'aspiration».

Genesis, vol. 11, no 1, p. 81-105.

SÉVIGNÉ (DE), Madame. 1973 [-1978]. Correspondance, édition de Roger Duchêne. Paris : Gallimard,

«Bibliothèque de la Pléiade », $3 \mathrm{t}$.

Sterne, Laurence. 2004 [1762]. La Vie et les opinions de Tristram Shandy, gentilhomme, traduction, préface et notes de Guy Jouvet. Auch (France) : Tristram, 937 p.

THÉVOz, Michel. 1989. Détournement d'écriture. Paris : Minuit, 119 p.

VALÉRY, Paul. 1936. Pièces sur l'art, 3e édition, revue et augmentée. Paris : Gallimard, 313 p.

Voltaire. 1952 [1770]. Lettres inédites à son imprimeur Gabriel Cramer, avec une introd. et des notes par Bernard Gagnebin. Genève; Lille : Droz; Giard, 316 p.

WIESEL, Elie. 2007 [1958]. La nuit, nouv. éd., préface d'Élie Wiesel, avant-propos de François Mauriac. Paris :

Minuit, «Double », $192 \mathrm{p}$. 